\title{
Stronger misdirection in curved than in straight motion
}

\author{
Jorge Otero-Millan ${ }^{1,2}$, Stephen L. Macknik ${ }^{1,3}$, Apollo Robbins ${ }^{4}$, Michael McCamy ${ }^{1}$ and Susana \\ Martinez-Conde ${ }^{1 *}$
}

${ }^{1}$ Neurobiology, Barrow Neurological Institute, Phoenix, AZ, USA

2 Signal Theory and Communications, University of Vigo, Vigo, Spain

${ }^{3}$ Neurosurgery, Barrow Neurological Institute, Phoenix, AZ, USA

${ }^{4}$ Whizmob Inc., Las Vegas, NV, USA

\section{Edited by:}

Luis M. Martinez, Universidad Miguel Hernández de Elche, Spain

\section{Reviewed by:}

Luis M. Martinez, Universidad Miguel Hernández de Elche, Spain

Peter Thompson, University of York, UK

\section{*Correspondence:}

Susana Martinez-Conde, Laboratory of Visual Neuroscience, Division of Neurobiology, Barrow Neurological Institute, 350 West Thomas Road,

Phoenix, AZ 85013, USA.

e-mail:smart@neuralcorrelate.com
Illusions developed by magicians are a rich and largely untapped source of insight into perception and cognition. Here we show that curved motion, as employed by the magician in a classic sleight of hand trick, generates stronger misdirection than rectilinear motion, and that this difference can be explained by the differential engagement of the smooth pursuit and the saccadic oculomotor systems. This research exemplifies how the magician's intuitive understanding of the spectator's mindset can surpass that of the cognitive scientist in specific instances, and that observation-based behavioral insights developed by magicians are worthy of quantitative investigation in the neuroscience laboratory.

Keywords: saccades, smooth pursuit, magic, illusion, sleight of hand, eye movements

\section{INTRODUCTION}

Visual illusions developed by painters and sculptors have aided the understanding of important principles of visual perception. Likewise, cognitive illusions developed by magicians can reveal critical clues in cognitive processing (Kuhn et al., 2008b; Macknik et al., 2008). Centuries of informal but systematic research in magic theory have predated contemporary cognitive science concepts such as "change blindness" (Simons and Levin, 1998), "inattentional blindness" (Simons and Chabris, 1999), and "choice blindness" (Johansson et al., 2005). Magic remains a rich and largely untapped source of insight into perception and cognition (Barnhart, 2010).

One of the authors (Apollo Robbins, The Gentleman Thief) is a professional magician who specializes in sleight of hand and stage pickpocketing. Apollo Robbins noticed that he could draw a spectator's attention in distinctive ways by moving his hands along different trajectories, for instance while secretly stealing an object from a "mark," or victim. Specifically, Apollo Robbins moves his hands in a curved motion to engage the spectator's attention along the motion trajectory, whereas he uses linear motion to shift attention from the start to the endpoint of a vector. Both types of movements decrease the attentional focus at the onset position of the hand movement, but with curvilinear motion the shift toward the final position is more permanent.

The French Drop is a classic sleight of hand magic trick (Figure 1; Movies S1 and S2 in Supplementary Material), with the following sequence: (a) The magician shows a coin or another small object between the fingers and thumb of one hand (i.e., left hand). (b) The right hand approaches the left hand and appears to take the coin. (c) The right hand moves away from the left hand as if carrying the coin; and (d) the magician opens his right hand to reveal that the coin has disappeared. This simulated maneuver results in the perception that the coin has magically vanished from the right hand (whereas in reality, it was not removed from the left).
Step (c) of the French Drop can be performed using either curved or straight hand motion. Although the illusion is effective either way, Apollo Robbins predicted that straight motion should result in the spectator's gaze bouncing from the open right hand back to the closed left hand (which retained the hidden coin) immediately after the reveal, whereas curved motion would cause the spectator's gaze to remain focused on the final hand rather than returning to the original hand. If true, the use of curved hand motions in certain magic routines may help to disrupt the "reconstruction process", that is, the ability of the spectator to reconstruct the trick after the performance, or to determine the secret method and link it to the intended magical effect.

\section{MATERIALS AND METHODS SUBJECTS}

Seven subjects (four females, three males) with normal or corrected-to-normal vision participated in this study. All subjects were naïve and were paid $\$ 15$ for a single experimental session. Experiments were carried out under the guidelines of the Barrow Neurological Institute's Institutional Review Board (protocol 04BN039), and written informed consent was obtained from each participant.

\section{EYE MOVEMENTS RECORDINGS AND ANALYSES}

Eye position was recorded non-invasively in both eyes with a fast video-based eye movement monitor (EyeLink 1000, SR Research) at 500 samples per second (instrument noise $0.01 \mathrm{rms}$ ).

We identified and removed blink periods as the portions of the EyeLink 1000 recorded data where the pupil information was missing. We added $200 \mathrm{~ms}$ before and after each period to further eliminate the initial and final parts of the blink, where the pupil is partially occluded. We moreover removed those portions of the data corresponding to very fast decreases and increases in pupil 


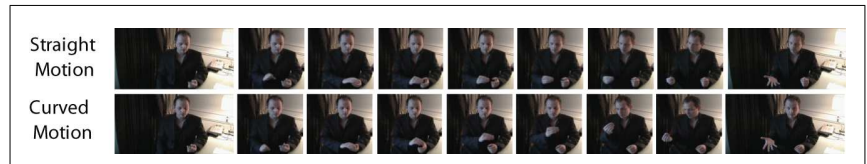

FIGURE 1 | Subset of frames from the straight and curved motion videos (see also Movies S1 and S2 in Supplementary Material).

area (>20 units per sample) plus the $200 \mathrm{~ms}$ before and after. Such periods are probably due to partial blinks, where the pupil is never fully occluded (thus failing to be identified as a blink by EyeLink; Troncoso et al., 2008).

We identified saccades with an objective algorithm (Engbert and Kliegl, 2003; $\lambda=6$ ). To reduce the amount of potential noise, we analyzed only binocular saccades (that is, saccades with a minimum overlap of one data sample in both eyes). Additionally, we imposed a minimum intersaccadic interval of $20 \mathrm{~ms}$ so that overshoot corrections were not categorized as saccades.

To identify pursuit we found all intersaccadic intervals longer than $80 \mathrm{~ms}$ and calculated the mean eye movement velocity in each of those periods, discarding the first $30 \mathrm{~ms}$ (to avoid interference from the preceding saccade and its overshoot). Pursuit periods were defined as those with a mean eye movement speed higher than $4 \%$ s. Trials with pursuit needed to contain at least one pursuit period.

We considered that subjects looked back to the original hand in any given trial if their gaze entered a $150 \times 100$ pixels box centered around the original hand for at least one data sample, after the reveal (i.e., the opening of the final hand).

To obtain the colormaps in Figure 2, we added the amount of time that subjects allocated their gaze to every pixel on the screen. Colormaps were smoothed with a Gaussian filter with a SD of 8 pixels.

\section{EXPERIMENTAL DESIGN}

Subjects rested their head on a chin/forehead-rest $57 \mathrm{~cm}$ from a video monitor (Barco Reference Calibrator V, 60- $\mathrm{Hz}$ refresh rate). Each experimental session included 4 blocks of 4 experimental conditions, for a total of 16 trials. The four experimental conditions were: curved motion without reveal, straight motion without reveal, curved motion with reveal and straight motion with reveal. In the conditions without reveal, the video clips stopped shortly before the magician opened his final hand. In each block, the first two trials corresponded to the two conditions without reveal, in random order, and the last two trials corresponded to the two conditions with reveal, also in random order. Subjects were asked to answer "where the coin was" after each of the trails without reveal, and "how did he (the magician) do it" after each of the trials with reveal.

Because an actual magician (i.e., rather than a cartoon or computer simulation) performed all maneuvers, motion features such as timing, duration, length, etc. could not be exactly equated across experimental conditions. Future research using computer simulations of the magician's hand movements should quantify the importance of the type of motion performed (i.e., rectilinear versus curvilinear) versus other motion parameters such as timing and duration.

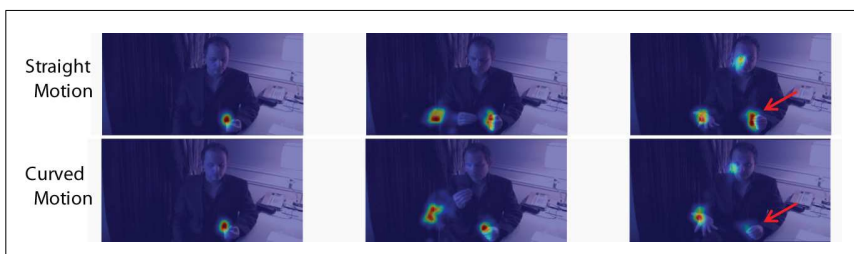

FIGURE 2 | Average eye positions of all subjects for three different video clip segments: before the right hand pretends to grab the coin, during the movement of the right hand, and after the right hand stops.

\section{RESULTS}

We tracked the eye movements of naïve subjects as they viewed videos of Apollo Robbins executing the French Drop with linear versus curved motion (Figure 1; Movies S1 and S2 in Supplementary Material). As predicted by Apollo Robbins, subjects showed different eye movement patterns for the two types of motion. The spectators' gaze stayed on the right hand more often after the curved motion, whereas it jumped back to the left hand after the straight motion (Figure 2; Movies S3 and S4 in Supplementary Material). Thus magicians manipulate not only the audience's gaze position during a sleight, but also the subsequent gaze location once the sleight is complete.

We tested if these effects could be due to differential engagement of the smooth pursuit versus the saccadic oculomotor systems (Macknik et al., 2008). Straight hand motion could invoke a saccadic eye movement. If so, suppression of visual perception during the saccade could result in reduced attention to the motion trajectory, leaving the attentional focus on the initial and/or final hand locations. Conversely, curvilinear motion might draw the spectator's oculomotor system into a long pursuit of the magician's wandering hand; in such case the retinal fovea would track the hand's non-linear trajectory, helping to draw the attentional spotlight along with it. Our results show that the spectators' oculomotor behavior is indeed different for both types of motion, with smooth pursuit being predominant in the curved motion condition, and saccades dominating in the straight motion condition. Further, spectators looked back less at the initial hand in trials containing smooth pursuit than in trials without smooth pursuit, irrespective of whether the hand moved in a straight or a curved path (Figure 3). None of these results were affected by training (i.e., the first and last trials offered comparable results; data not shown).

The subjects' verbal responses did not differ across conditions (data not shown), possibly because subjects were queried immediately after the vanish, while the last frame of the video clip in question remained visible, or because the trick was presented in isolation, rather than as part of a magic routine (an arrangement of tricks organized in logical fashion as part of a magic performance).

\section{DISCUSSION}

Our results indicate that curvilinear motion is a more powerful source of misdirection than rectilinear motion, as used in a classical sleight of hand trick. Particularly, the use of curvilinear motion in the simulated maneuver at the core of the French Drop sleight prevented observers from looking back at the hand that actually retained the coin - after the magician revealed that the 

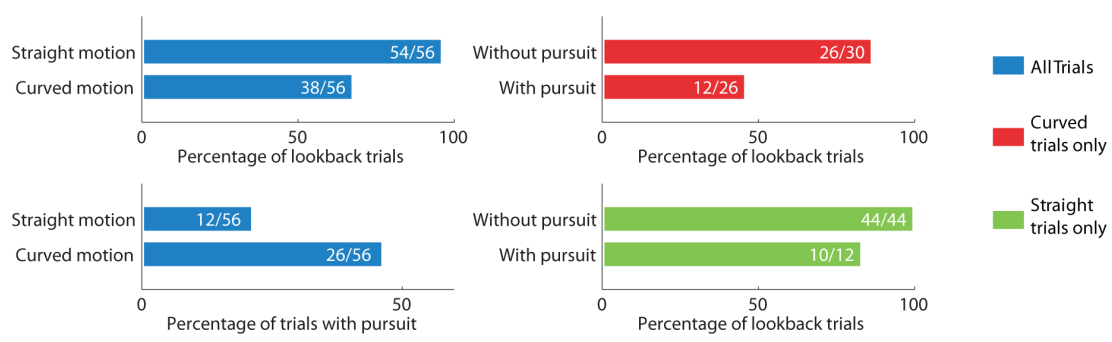

FIGURE 3 | Subjects looked back to the original hand more often in straight than in curved motion trials $(p=0.0001)$. Smooth pursuit predominated in curved motion trials $(p=0.009)$. Subjects ignored the original hand when smooth pursuit was present, both in curved ( $p=0.001)$ and straight ( $p=0.04)$ motion trials. $p$ Values were calculated using the Fisher exact test. The numbers of trials used to calculate the percentages are indicated inside the bars. hand that had appeared to take the coin was empty (Figure 2). To our knowledge, this is the first observation by a non-scientist member of the magic community to have led to a previously unknown, neuroscientific discovery.

Our data moreover show that the differences in the observers' gaze position at the end of each trial are strongly dependent on the presence or absence or pursuit eye movements during the viewing of the sleight, with curvilinear trials typically generating pursuit eye movements more often than rectilinear trials (Figure 3 ). This suggests a differential engagement of the smooth pursuit and saccadic systems in the dynamic control of attentional focus.

Previous work has investigated the magicians' use of social misdirection cues, such as their own gaze direction, to manipulate the audience's eye position (Kuhn and Tatler, 2005; Kuhn and Land, 2006; Kuhn et al., 2008a; Kuhn and Findlay, 2010; Cui et al., 2011). Here we show for the first time that different types of hand motion, as used by magicians, can have differential effects on the oculomotor behavior of observers.

Curvilinear target motions may be more salient intrinsically than linear target motions (in addition to the two types of motion affecting differentially the oculomotor system) (Kristjánsson and Tse, 2001). In the spatial domain, the curves and the corners of object surfaces are perceptually more salient and generate stronger neural activity than straight edges, possibly owing to the fact that they are less redundant and predictable, and therefore more informative (Troncoso et al., 2005, 2007, 2009). The same redundancy reduction argument might apply to non-predictable objectmotion trajectories, such as curvilinear versus straight motion. If this is the case, curvilinear motion trajectories should be more salient (and consequently engage stronger attention) than straight trajectories.

The capacity of curvilinear movement to misdirect the gaze and/or the attention of observers along a motion trajectory may

\section{REFERENCES}

Barnhart, A. S. (2010). The exploitation of Gestalt principles by magicians. Perception 39, $1286-1289$.

Cui, J., Otero-Millan, J., Macknik, S. L., King, M., and Martinez-Conde, S. (2011). Social misdirection fails to enhance a magic illusion.

have far reaching implications outside of magic and pickpocketing, such as in the application of predator-evasion strategies in the natural world, in military tactics, in sports misdirection, and in marketing. Our results demonstrate that magic theory can provide new windows into the psychological and neural principles of perception and cognition.

\section{CONCLUSION}

We show that curved motion, as used in a classical sleight of hand trick, is a more powerful source of magic misdirection than straight motion, and that the difference can be explained by the differential engagement of the smooth pursuit and the saccadic oculomotor systems - with curvilinear trials generating pursuit eye movements more often than rectilinear trials. These findings may have far reaching implications beyond magic, such as in the application of predator-evasion strategies in the natural world, in military tactics, in sports misdirection, and in marketing. This research also demonstrates that magic theory can provide new windows into the psychological and neural principles of perception and cognition; thus behavioral insights developed by magicians are worthy of quantitative investigation in the laboratory.

\section{ACKNOWLEDGMENTS}

We thank Andrew Danielson for technical assistance. This study was supported by the Barrow Neurological Foundation (Stephen L. Macknik and Susana Martinez-Conde) and the National Science Foundation (award 0852636 to Susana Martinez-Conde). Jorge Otero-Millan is a Fellow of the Pedro Barrié de la Maza Foundation.

\section{SUPPLEMENTARY MATERIAL}

The Supplementary Material for this article can be found online at http://www.frontiersin.org/human_neuroscience/10.3389/fnhum. 2011.00133/abstract

outcome in a simple decision task. Science 310, 116-119.

Kristjánsson, A., and Tse, P. U. (2001). Curvature discontinuities are cues for rapid shape analysis. Percept. Psychophys. 63 390-403.

Kuhn, G., and Findlay, J. M. (2010). Misdirection, attention and awareness: inattentional blindness reveals temporal relationship between eye movements and visual awareness. Q. J. Exp. Psychol. 63, 136-146.

Kuhn, G., and Tatler, B. W. (2005). Magic and fixation: now you don't see it, now you do. Perception 34, 1155-1161. 
Kuhn, G., Tatler, B. W., Findlay, J. M., and Cole, G. G. (2008a). Misdirection in magic: implications for the relationship between eye gaze and attention. Vis. Cogn. 16, 391-405.

Kuhn, G., Amlani, A. A., and Rensink, R. A. (2008b). Towards a science of magic. Trends Cogn. Sci. (Regul. Ed.) $12,349-354$.

Kuhn, G. L. M. F., and Land, M. F. (2006). There's more to magic than meets the eye. Curr. Biol. 16, 950.

Macknik, S. L., King, M., Randi, J., Robbins, A., Teller, T., Thompson, J., and Martinez-Conde, S. (2008). Attention and awareness in stage magic: turning tricks into research. Nat. Rev. Neurosci. 9, 871-879.
Simons, D. J., and Chabris, C. F. (1999). Gorillas in our midst: sustained inattentional blindness for dynamic events. Perception 28, 1059-1074.

Simons, D. J., and Levin, D. T. (1998). Failure to detect changes to people during a real-world interaction. Psychon. Bull. Rev. 5, 644-649.

Troncoso, X. G., Macknik, S. L., and Martinez-Conde, S. (2005). Novel visual illusions related to Vasarely's "nested squares" show that corner salience varies with corner angle. Perception 34, 409-420.

Troncoso, X. G., Macknik, S. L., and Martinez-Conde, S. (2009). Corner salience varies linearly with corner angle during flicker-augmented contrast: a general principle of corner perception based on
Vasarely's artworks. Spat. Vis. 22, 211-224.

Troncoso, X. G., Macknik, S. L., OteroMillan, J., and Martinez-Conde, S. (2008). Microsaccades drive illusory motion in the Enigma illusion. Proc. Natl. Acad. Sci. U.S.A. 105, 16033-16038.

Troncoso, X. G., Tse, P. U., Macknik, S. L., Caplovitz, G. P., Hsieh, P.-J., Schlegel, A. A., OteroMillan, J., and Martinez-Conde, S. (2007). BOLD activation varies parametrically with corner angle throughout human retinotopic cortex. Perception 36, 808-820.

Conflict of Interest Statement: The authors declare that the research was conducted in the absence of any commercial or financial relationships that could be construed as a potential conflict of interest.

Received: 10 April 2011; accepted: 24 October 2011; published online: 21 November 2011.

Citation: Otero-Millan J, Macknik SL, Robbins A, McCamy $M$ and MartinezConde S (2011) Stronger misdirection in curved than in straight motion. Front. Hum. Neurosci. 5:133. doi: 10.3389/fnhum.2011.00133

Copyright $\odot 2011$ Otero-Millan, Macknik, Robbins, McCamy and MartinezConde. This is an open-access article subject to a non-exclusive license between the authors and Frontiers Media SA, which permits use, distribution and reproduction in other forums, provided the original authors and source are credited and other Frontiers conditions are complied with. 